\title{
The diagnostic value of parathyroid hormone washout after fine- needle aspiration in patients with primary hyperparathyroidism
}

\author{
${ }^{1}$ Fotini Adamidou, ${ }^{1}$ Panagiotis Anagnostis, ${ }^{1}$ Champidis Vasileios, ${ }^{1}$ Simoni Katergari, \\ 2Zacharioudakis Georgios, 'Marina Kita
}

\author{
${ }^{1}$ Department of Endocrinology and Diabetes, Hippokration General Hospital, Thessaloniki, Greece \\ ${ }^{2}$ Fifth Department of Surgery, Aristotle University of Thessaloniki, Hippokration General Hospital, Thessaloniki, Greece
}

\section{Introduction}

Commonly used pre-operative methods (ultrasonography and sestamibi scan) to localize the culprit parathyroid gland(s) in patients with primary hyperparathyroidism (PHPT), frequently yield false positive or false negative results

Parathyroid hormone (PTH) washout after fineneedle aspiration (FNA) may allow for a targeted surgical approach.

\section{Aims}

The aim of this study was to test the diagnostic value of preoperative PTH washout after FNA in PHPT patients.

\section{Methods/design}

Retrospective study conducted at the department of Endocrinology in Hippokration General Hospital (Thessaloniki, Greece), a tertiary referral centre for endocrinology and diabetes.

Ultrasound-guided FNA was performed if a structure compatible with a parathyroid adenoma was found on cervical ultrasound.

A PTH washout cut-off value was considered diagnostic if it was higher than the concomitant serum PTH value.

\section{Results}

29 patients [2 (6.8\%) males, mean age $58.6 \pm 9.1$ years] were included.

A single adenoma was identified in $26(89.7 \%)$ patients and a double adenoma in $1(3.4 \%)$.

$2(6.9 \%)$ patients were harbouring an ectopic parathyroid adenoma (upper mediastinal and intrathyroid).

24 patients $(82.7 \%)$ had elevated PTH washout concentrations

In 10 out of 12 patients with a negative sestamibi scan, the PTH washout yielded a truly positive result.

\begin{tabular}{|c|c|c|}
\hline Variable & Mean values & $\begin{array}{l}\text { Reference } \\
\text { range }\end{array}$ \\
\hline $\begin{array}{l}\text { Pre-operative PTH } \\
\text { (pg/mL) }\end{array}$ & $194.2 \pm 176.8$ & $10-53$ \\
\hline $\begin{array}{l}\text { Pre-operative total } \\
\text { serum calcium } \\
\text { (mg/dL) }\end{array}$ & $11.5 \pm 1.4$ & $8.8-10.6$ \\
\hline $\begin{array}{l}\text { Pre-operative } \\
25(\mathrm{OH}) \mathrm{D}(\mathrm{ng} / \mathrm{mL})\end{array}$ & $24.1 \pm 20.5$ & $>30$ (sufficiency) \\
\hline Post-operative PTH & $45.9 \pm 26.3$ & $10-53$ \\
\hline $\begin{array}{l}\text { Post-operative total } \\
\text { serum calcium }\end{array}$ & $9.04 \pm 0.7$ & $8.8-10.6$ \\
\hline PTH washout & $2009.5 \pm 1417.6$ & $\begin{array}{l}\text { >upper serum } \\
\text { levels }\end{array}$ \\
\hline
\end{tabular}

\section{Diagnostic value of PTH-washout}

True positive 24

True negative 4

Sensitivity $85.7 \%$

Specificity $100 \%$

Positive predictive value $100 \%$

\section{Conclusions}

Noussios G, Anagnostis P, et al. Exp Clin Endocrinol Diabetes 2012;120:604-10 Abdelghani $R$ et al. Laryngoscope 2013;123:1310-3

Bancos I, et al. Endocr Pract. $2012 ; 18: 441-9$

Elevated PTH washout concentrations after ultrasoundguided FNA is an highly accurate diagnostic tool in identifying parathyroid adenomas and guiding parathyroid surgery. 